\title{
Long non-coding RNA Igf2as controls hepatocellular carcinoma progression through the ERK/MAPK signaling pathway
}

\author{
HAN BAO $^{1 *}$, CHUN-GUANG GUO ${ }^{2 *}$, PENG-CHENG QIU ${ }^{1}$, XIN-LEI ZHANG ${ }^{1}$, QI DONG ${ }^{3}$ and YU-KUN WANG ${ }^{1}$ \\ ${ }^{1}$ Department of Pharmacy Administration and Drug Information, School of Pharmacy, \\ Fourth Military Medical University, Xi'an, Shaanxi 710032; ${ }^{2}$ Department of Second Hepatobiliary \\ Surgery, The First People's Hospital of Xianyang City, Shaanxi 712000, P.R. China; ${ }^{3}$ Department of \\ Nano Fusion Technology, Pusan University, Miryang, Kyungnam 609-735, Republic of Korea
}

Received August 11, 2015; Accepted February 3, 2017

DOI: $10.3892 / 01.2017 .6492$

\begin{abstract}
Long non-coding RNAs (lncRNAs) serve an important role in numerous human diseases, including cancer. Abnormal expression of lncRNAs has been associated with a number of tumor types; however, the underlying mechanisms through which lncRNA functions have yet to be elucidated. The present study primarily focuses on insulin-like growth factor 2 antisense 1 (Igf2as), a lncRNA reported to be differentially expressed in hepatocellular carcinoma (HCC). Reverse transcription-quantitative polymerase chain reaction analysis was used to determine the level of Igf2as in HCC cells and tissues. Flow cytometry was used to determine the level of cell apoptosis following Igf2as suppression and western blot analysis was used to identify altered protein expression levels. The results demonstrated that Igf2as was upregulated in $\mathrm{HCC}$ cells and tissues, and that the inhibition of Igf2as using a targeted small interfering RNA (si-Igf2as), significantly decreased cell proliferation and increased apoptosis. Western blot analysis identified that the extracellular signal-regulated kinase/mitogen-activated protein kinase (ERK/MAPK) signaling pathway was inhibited in cells transfected with si-Igf2as. In addition, cell migration was markedly reduced by the knockdown of Igf2as. These results suggest that lncRNA Igf2as may control hepatocellular progression primarily through the regulation of the ERK/ MAPK signaling pathway.
\end{abstract}

Correspondence to: Professor Yu-Kun Wang, Department of Pharmacy Administration and Drug Information, School of Pharmacy, Fourth Military Medical University, 169 Changle West Road, Xi'an, Shaanxi 710032, P.R. China

E-mail:wangyk@fmmu.edu.cn

*Contributed equally

Key words: Igf2as, hepatocellular carcinoma, extracellular signal-regulated kinases/mitogen-activated protein kinase, cell proliferation

\section{Introduction}

Hepatocellular carcinoma (HCC) is one of the most prevalent malignancies globally (1). In China, it is a major cause of adult mortalities (2). It has been reported that hepatitis, non-alcoholic fatty liver disease and alcohol abuse are the primary contributors to HCC (3). In the majority of circumstances, the treatment that is most likely to be curative is a liver transplant following surgical tumor resection. However, the frequency of tumor metastasis and recurrence are important risk factors in the long-term survival of patients with HCC (4). Therefore, it is important to investigate the specific mechanisms underlying the pathology of HCC.

Non-coding RNAs (ncRNAs) have been established to be important for tumor cell proliferation, migration, invasion, apoptosis, angiogenesis and drug resistance, despite previously being considered as 'transcriptional noise' (5-7). Genomic studies have identified that only $\sim 2 \%$ of all genes encode proteins (8). ncRNAs may be divided into microRNAs (miRNAs) and long non-coding RNAs (lncRNAs), which are $>200$ nucleotides (nt) long, and have been established to serve important roles in a number of cellular functions (9). At present, the abnormal expression of lncRNAs has been identified to cause tumor-suppressive and pro-tumor effects in different types of cancer $(7,10)$. For instance, antisense non-coding RNA in the INK4 locus (ANRIL) lncRNA has been reported to be increased in the HCCs and associated with poor prognosis (11). In addition, sex-determining region Y-Box overlapping transcript lncRNA was determined to be upregulated in HCC and enhance cancer cell metastasis (12).

In the current study, the primary focus was the IncRNA Igf2as, which is transcribed from the IGF2 region in a reverse orientation. In prostate cancer, Igf2as was identified to be upregulated and significantly enhance cancer cell proliferation, suggesting it serves an important role in the survival of patients with prostate cancer (13). Although, Igf2as was established to be overexpressed in Wilms' tumor, the epigenetically imprinted form of Igf2as was not identified (14). The present study investigated the role of Igf2as in HCC progression and demonstrated that the upregulation of Igf2as promotes HCC progression primarily through the regulation of the MAPK/ ERK signaling pathway. 


\section{Materials and methods}

Cell culture and transfection. HepG2, SMMC7721, Huh7 human HCC cells and the HL-7702 human immortalized normal hepatocyte cells purchased from American Type Culture Collection (Manassas, VA, USA) were cultured in Dulbecco's modified Eagle's medium supplemented with 10\% fetal bovine serum (both Gibco; Thermo Fisher Scientific, Inc., Waltham, MA, USA), streptomycin $(100 \mathrm{mg} / \mathrm{ml})$ and penicillin $(100 \mathrm{IU} / \mathrm{ml})$ at $37^{\circ} \mathrm{C}$ in a humidified atmosphere containing $5 \% \mathrm{CO}_{2}$.

HepG2 cells were transfected with siRNA targeting Igf2as (si-Igf2as) using HiPerFect Transfection Reagent (Qiagen China Co., Ltd., Shanghai, China) according to the manufacturer's protocol. si-Igf2as and the negative control (NC; no. A06001) were purchased from Shanghai Jima Pharmaceutical Technology, Co., Ltd. (Shanghai, China). The specific siRNA sequence for Igf2as was 5'-GUCAUCUCAGGUCAUUCUAU-3'.

Immunohistochemistry and evaluation. A total of 50 eligible patients were enrolled. There were $42(84 \%)$ males and 8 (16\%) females. The median age was 74 (range, 35-88) years. Samples of HCC tissues and corresponding benign liver tissues ( $3 \mathrm{~cm}$ away from the tumor) were obtained from patients who underwent curative surgery for HCC at Fourth Military Medical University Xijing Hospital (Xi'an, China) between March 2015 and March 2016. All HCC and normal liver tissue samples were pathologically confirmed by a hepatologist. Written informed consent for the biological studies was obtained from each patient involved in the study and the study was approved by the Ethics Committee of Xijing Hospital (Xi'an, China).

Briefly, tissue samples for immunohistochemistry were fixed with $10 \%$ formalin at room temperature for $\geq 24 \mathrm{~h}$ and embedded in paraffin. Sections $(3 \mathrm{~mm})$ from paraffin-embedded blocks were deparaffinized using xylene and rehydrated in a descending ethanol gradient $(99.5,90$, 70 and $50 \%$ ). Antigen retrieval was performed by heating the sections in an autoclave at $121^{\circ} \mathrm{C}$ for $10 \mathrm{~min}$ in $10 \mathrm{mM}$ citrate buffer. Endogenous peroxidase activity was blocked with $0.3 \%$ hydrogen peroxide for $10 \mathrm{~min}$ at room temperature. Following washing three times with PBS, the sections were incubated overnight at $4^{\circ} \mathrm{C}$ in a humidified chamber with a primary antibody directed against novel oncogene with kinase domain (NOK; no. QPREST28987; Sigma-Aldrich; Merck KGaA, Darmstadt, Germany) at a dilution of 1:200. The next day, following washing three times with PBS, the sections were incubated with mouse secondary antibody (dilution, 1:200; no. PV-9005; Beijing Zhongshan Golden Bridge Biotechnology Co., Ltd., Beijing, China) for $30 \mathrm{~min}$ at room temperature. Finally, the sections were stained with diaminobenzidine and the nucleus was counterstained with Mayer's hematoxylin. The negative control was treated with PBS instead of the primary antibody. The sections were evaluated under a microscope by two experienced pathologists who were blinded to the clinicopathological data of the tissue samples. Five fields were randomly selected and three slides from each tissue sample were analyzed. Scores were given based on the percentage of positively stained tumor cells $(0,0 \% ; 1,1-10 \%$;
$2,11-50 \% ; 3,51-80 \% ; 4,81-100 \%)$ and the staining intensity 0) negative; i) weak; ii) moderate; iii) strong). The numeric values of these two parameters were multiplied to obtain an immunoreactivity score (IRS) ranging from 0-12. For analysis, two groups with low (IRS 0-4) or high NOK IRS (IRS 6-12) expression levels were formed.

$R N A$ extraction and reverse transcription-quantitative polymerase chain reaction (RT- $q P C R)$ analysis. Total RNA was extracted from HCC tissues and HepG2, SMMC7721 and Huh7 human HCC cells using TRIzol reagent according to the manufacturer's protocol (Invitrogen; Thermo Fisher Scientific, Inc.). Subsequently, RNA was reverse transcribed into complementary DNA using the EasyScript One-Step gDNA Removal and cDNA Synthesis SuperMix kit (Beijing Transgen Biotech Co., Ltd., Beijing, China). Then, RT-qPCR was applied to quantify the level of Igf2as. Primers were as follows: IGF2as forward, 5'-CCTTGGACTTTGAGT CAAATT-3' and reverse, 5'-GGTCGTGCCAATTACATT TCA-3'; U6 forward, 5'-CTCGCTTCGGCAGCACA-3' and reverse, 5'-AACGCTTCACGAATTTGCGT-3'. All primers were synthesized by Shanghai Biological Engineering Co., (Shanghai, China). The thermocycling conditions were as follows: $95^{\circ} \mathrm{C}$ for $10 \mathrm{~min} ; 40$ cycles at $95^{\circ} \mathrm{C}$ for $15 \mathrm{sec}$; and $60^{\circ} \mathrm{C}$ for $1 \mathrm{~min}$. PCR amplification was performed using the Quant qRT-PCR kit SYBR Green kit (\#FP302-01; Tiangen Biotech Co., Ltd., Beijing, China) according to the manufacturer's protocol and reactions were analyzed using the ABI StepOne Plus System (Applied Biosystems; Thermo Fisher Scientific, Inc.). Relative IncRNA expression was normalized against the endogenous control, U6, using the $2^{-\Delta \Delta \mathrm{Cq}}$ method (15). Each experiment was performed three times and the average results were calculated.

Immunofluorescence assay. The HepG2 cells $\left(1 \times 10^{5}\right)$ were grown on cover slips for $24 \mathrm{~h}$ at $37^{\circ} \mathrm{C}$. Briefly, cells were fixed in $4 \%$ paraformaldehyde for $10 \mathrm{~min}$ at room temperature. Following the fixation, cover slips were washed with PBS. The cells were blocked with $5 \%$ bovine serum albunim (BSA) in PBS for $1 \mathrm{~h}$ at room temperature and then treated overnight at $4^{\circ} \mathrm{C}$ with anti-p-ERK antibody (no. M9692; 1:100 dilution; Sigma-Aldrich; Merck KGaA). Following incubation, cells were washed and treated with fluorescein isothiocyanate (FITC)-labeled secondary antibody (no. PV-9005; 1:200 dilution; Beijing Zhongshan Golden Bridge Biotechnology Co., Ltd.) for $1 \mathrm{~h}$ at room temperature in dark. Immunofluorescent images were captured and analyzed using the Olympus light microscope (Olympus Corporation, Tokyo, Japan).

Western blot analysis. Total protein was extracted from HepG2, SMMC7721 and Huh7 human HCC cells using radioimmunoprecipitation assay buffer (Beijing Solarbio Science \& Technology Co., Ltd., Beijing, China) supplemented with $1 \%(\mathrm{v} / \mathrm{v})$ phenylmethylsulfonyl fluoride (Beijing Solarbio Science \& Technology Co., Ltd.), 0.3\% (v/v) protease inhibitor (Sigma-Aldrich; Merck KGaA) and 0.1\% (v/v) phosphorylated proteinase inhibitor (Sigma-Aldrich; Merck KGaA). In total $15 \mathrm{mg}$ of protein was separated on a $10 \%$ gel using SDS-PAGE and transferred onto a polyvinylidene fluoride membrane. The membrane was incubated 
with $8 \%$ milk for $2 \mathrm{~h}$ at room temperature. Following three washes with PBS-Tween-20 (PBST; $5 \mathrm{~min} /$ wash), the membrane was incubated with the primary antibodies ERK (dilution, 1:2,000; no. M3807; Sigma-Aldrich; Merck KGaA), phosphorylated-ERK (p-ERK; dilution, 1:2,000; no. M9692; Sigma-Aldrich; Merck KGaA), protein kinase B (Akt; dilution, 1:1,500; no. SAB1400006; Sigma-Aldrich; Merck KGaA), phosphorylated-Akt (p-Akt; dilution, 1:1,000; no. SAB4301497; Sigma-Aldrich; Merck KGaA), B-cell lymphoma 2 (Bcl-2; dilution, 1:1,000; no. B3170; Sigma-Aldrich; Merck KGaA), Snaill (dilution, 1:2,000; no. SAB2108482; Sigma-Aldrich; Merck KGaA) and $\beta$-actin (dilution, 1:2,000; no. A5441; Sigma-Aldrich; Merck KGaA) at $4^{\circ} \mathrm{C}$ overnight. Then, the membranes were washed with PBST three times and incubated with the horseradish peroxidase (HRP)-conjugated secondary antibody (dilution, 1:2,000; no. ZDR-5301; Beijing Zhongshan Golden Bridge Biotechnology Co., Ltd.) at room temperature for $2 \mathrm{~h}$. The membrane was washed three times with TBST, each for 5 min and detected using the ECL Western Blot kit (Beijing CoWin Biotech Co., Ltd., Beijing, China) according to the manufacturer's protocol. Then, the relative band density was determined using enhanced chemiluminescence and quantified using Sigmatel software v2.0 (Jandel Scientific Software, San Rafael, CA, USA). $\beta$-actin was used as the internal control.

MTT assay. Prior to the MTT assay, HepG2 cells were transfected with si-Igf2as or NC and cultured for 24,48 or $72 \mathrm{~h}$. The cells were collected and washed with PBS three times $(5 \mathrm{~min} /$ wash). Then, $5 \mathrm{mg} / \mathrm{ml}$ MTT reagent was added to each well and the cells were incubated for $4 \mathrm{~h}$ at $37^{\circ} \mathrm{C}$. The formazan crystals that formed were dissolved in $200 \mathrm{ml}$ dimethylsulfoxide and the absorbance was measured at a wavelength of $570 \mathrm{~nm}$. Each assay was performed three times and the average results were calculated.

Quantification of apoptotic cells. An Annexin-V FITC-propidium iodide (PI) Apoptosis kit (Invitrogen: Thermo Fisher Scientific, Inc.) was used to determine the level of apoptosis. HepG2 cells were transfected with si-Igf2as and cultured for $48 \mathrm{~h}$ prior to the assay. These cells were collected and washed with PBS three times. The cells were resuspended in ice-cold $95 \%$ ethanol with $0.5 \%$ Tween 20 . The fixed cells were washed in $1 \%$ BSA-PBS solution. Then, the cells were resuspended in 1X Annexin-V binding buffer [10 mM HEPES/ $\mathrm{NaOH}$ (pH 7.4), $140 \mathrm{mM} \mathrm{NaCl}, 2.5 \mathrm{mM} \mathrm{CaCl}{ }_{2}$ ]. Annexin-V FITC and PI were added to the cells at room temperature for $15 \mathrm{~min}$ in the darkness. Following incubation, the cells were filtered and analyzed using a flow cytometer (BD Biosciences, Franklin Lakes, NJ, USA) within $1 \mathrm{~h}$ of staining. The number of cells in each quadrant was expressed as percentages of total stained cells. Data from a minimum of three independent repeats was used to quantify the number of apoptotic cells and graphed with GraphPad Prism 7 software (GraphPad Software, Inc., La Jolla, CA, USA).

5-ethynyl-2'-deoxyuridine (EdU) incorporation assay. HepG2 cells were transfected with si-Igf2as and cultured for $48 \mathrm{~h}$ prior to the assay. The EdU incorporation assay was performed using
Cell-Light ${ }^{\mathrm{TM}}$ EdUTP Apollo ${ }^{\circledR} 567$ TUNEL Cell Detection kit (\#C10812-1; Guangzhou RiboBio Co., Ltd., Guangzhou, China) according to the manufacturer's protocol. For Hoechst staining, the cells were harvested onto a clean, fat-free glass slide at room temperature with a cytocentrifugation at $800 \mathrm{xg}$ for $10 \mathrm{~min}$. They were stained in $4 \mu \mathrm{g} / \mathrm{ml}$ Hoechst 33342 (\#H342; Dojindo Molecular Technologies, Inc., Shanghai, China) for $10 \mathrm{~min}$ at $37^{\circ} \mathrm{C}$ in darkness and washed twice in PBS. The slides were mounted with glycerol and observed using the Olympus optical photomicroscope. The samples were observed and photographed using the Olympus optical photomicroscope (Olympus Corporation). The percentage of EdU positive cells was calculated as follows: (EdU intensity in cells/Hoechst stained cells) x100.

Cell migration and invasion assay. Following transfection with si-Igf 2 as or $\mathrm{NC}$ for $48 \mathrm{~h}$, the cells (density, $5 \times 10^{4}$ ) were placed into the upper layer of a chamber (pore size, $8.0 \mu \mathrm{m}$; EMD Millipore, Billerica, MA, USA) for the migration assay. For the invasion assay, Matrigel (Sigma-Aldrich; Merck $\mathrm{KGaA}$ ) was pre-coated onto the upper chamber and cells were seeded at a density of $1 \times 10^{5}$, following transfection with si-Igf2as or NC for $48 \mathrm{~h}$. Then, the cells on the upper surface were fixed with $4 \%$ formaldehyde and stained with $0.05 \%$ crystal violet for $2 \mathrm{~h}$, and the cells were observed using a microscope.

Colony formation assay. The HepG2 and Huh7 cells (500 cell/well) were seeded separately in $60 \mathrm{~mm}$ dish at $24 \mathrm{~h}$ post-transfection and cultured for 10 days at $37^{\circ} \mathrm{C}$. Only colonies which reached $>50$ cells were fixed with $10 \%$ formaldehyde for $15 \mathrm{~min}$ and dyed with $8.0 \%$ crystal violet for $15 \mathrm{~min}$ at room temperature. Each experiment was repeated three times.

Statistical analysis. The data were expressed as the mean \pm standard error of the mean. Multiple comparisons were performed using one-way analysis of variance followed by Tukey's range test. SPSS Statistics v19 software was used for statistical analysis (IBM SPSS, Armonk, NY, USA). $\mathrm{P}<0.05$ was considered to indicate a statistically significant difference.

\section{Results}

Overexpression of Igf2as in HCC tissues and hepatoma cells. The present study examined the expression of Igf2as in HCC tissues and cells. As presented in Fig. 1A, Igf2as was significantly increased in HCC tissues compared with normal tissue $(\mathrm{P}<0.01)$. Similarly, RT-qPCR analysis demonstrated that the relative level of Igf2as was significantly increased in HepG2, SMMC7721 and Huh7 human HCC cells compared with the HL-7702 human immortalized hepatocyte cells $(\mathrm{P}<0.01$, $\mathrm{P}<0.05$ and $\mathrm{P}<0.05$, respectively; Fig. 1B).

Inhibition of Igf2as decreases HepG2 cell viability and promotes cell apoptosis. To explore the role of Igf2as, a specific siRNA targeting Igf2as was used. Igf2as was significantly inhibited in cells transfected with si-Igf2as for 24, 48 and $72 \mathrm{~h}$ $(\mathrm{P}<0.01, \mathrm{P}<0.001$ and $\mathrm{P}<0.001$, respectively; Fig. 2A). Notably, 
A

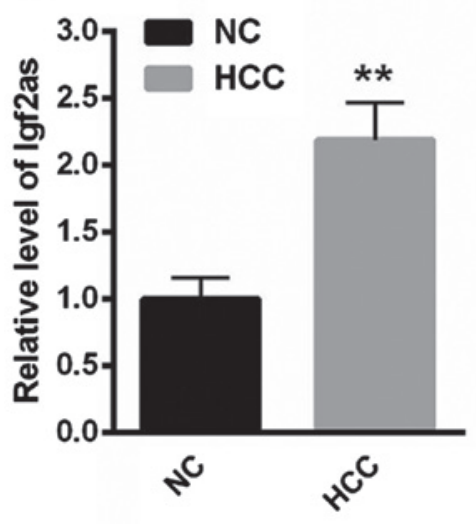

B

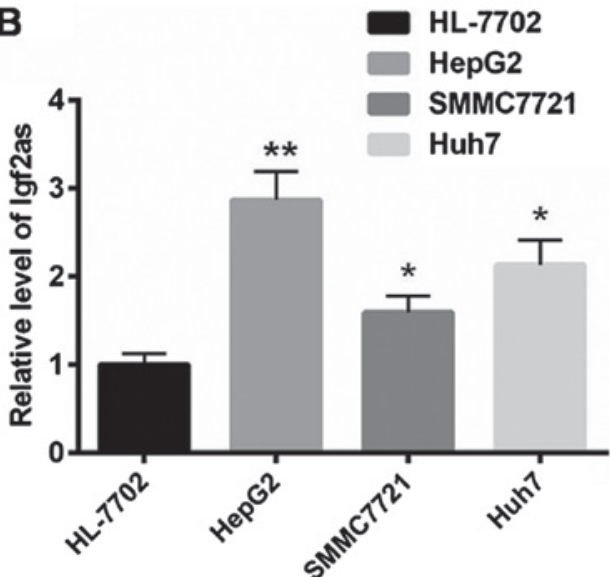

Figure 1. Igf2as expression is significantly increased in (A) HCC tissues and (B) hepatoma cells. ${ }^{*} \mathrm{P}<0.05,{ }^{* *} \mathrm{P}<0.01$ vs. NC tissue or HL-7702 cells. HCC, hepatocellular carcinoma; Igf2as, insulin-like growth factor 2 antisense 1 ; NC, negative control.

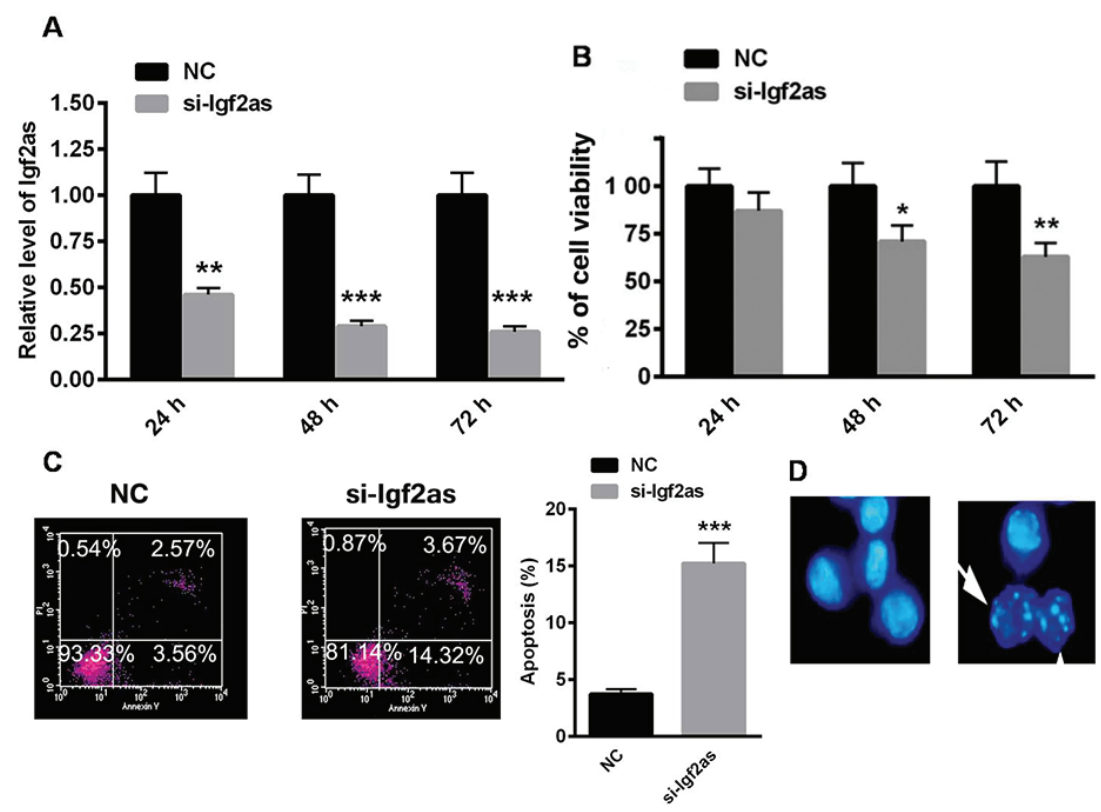

Figure 2. Inhibition of Igf2as decreases HepG2 cell viability and induces cell apoptosis. (A) Igf2as was significantly inhibited in cells transfected with si-Igf2as for 24,48 and $72 \mathrm{~h}$. (B) Inhibition of Igf2as decreased cell viability in a time-dependent manner, demonstrated by an MTT assay. Enhanced cell apoptosis occurred when HepG2 cells were transfected with an siRNA targeting Igf2as as demonstrated with (C) flow cytometry and (D) Hoechst nuclear staining. ${ }^{*} \mathrm{P}<0.05,{ }^{* *} \mathrm{P}<0.01,{ }^{* * * *} \mathrm{P}<0.001$ vs. the NC. Igf2as, insulin-like growth factor 2 antisense 1; NC, negative control; siRNA, small interfering RNA; si-Igfas, siRNA targeting insulin-like growth factor 2 antisense 1.

the knockdown of Igf2as decreased HepG2 cell viability in a time-dependent manner, as demonstrated with the MTT assay (Fig. 2B). Flow cytometry demonstrated that the cell apoptosis rate was significantly enhanced following transfection with si-Igf 2 as for 48 h $(\mathrm{P}<0.001$; Fig. $2 \mathrm{C})$. Hoechst staining also identified apoptotic HepG2 cells following transfection with si-Igf2as (Fig. 2D).

Suppression of Igf2as reduces colony formation and cell migration in HCC cells. When Igf2as was inhibited in HepG2 and Huh7 cells, colony formation capacity was markedly decreased (Fig. 3A). In addition, knockdown of Igf2as also reduced HepG2 and Huh7 cell migration ability (Fig. 3B). EdU incorporation was also decreased in cells transfected with si-Igf2as compared with the NC cells $(\mathrm{P}<0.01$; Fig. $3 \mathrm{C})$ suggesting reduced cell proliferation capacity.

Positive correlation between Igf2as expression levels and ERK activation. The activation of ERK in HCC tissues was investigated (Fig. 4). As presented in Fig. 4A, the phosphorylation level of ERK was notably increased in HCC tissues. By contrast, when Igf2as was inhibited, the level of phosphorylated ERK was markedly reduced (Fig. 4B). The protein expression levels of a number of ERK-associated proteins were evaluated in cells transfected with si-Igf2as. Western blot analysis demonstrated that ERK activation was significantly decreased in cells transfected with si-Igf2as $(\mathrm{P}<0.01$; Fig. 4C and D). Additionally, downstream signaling, including 

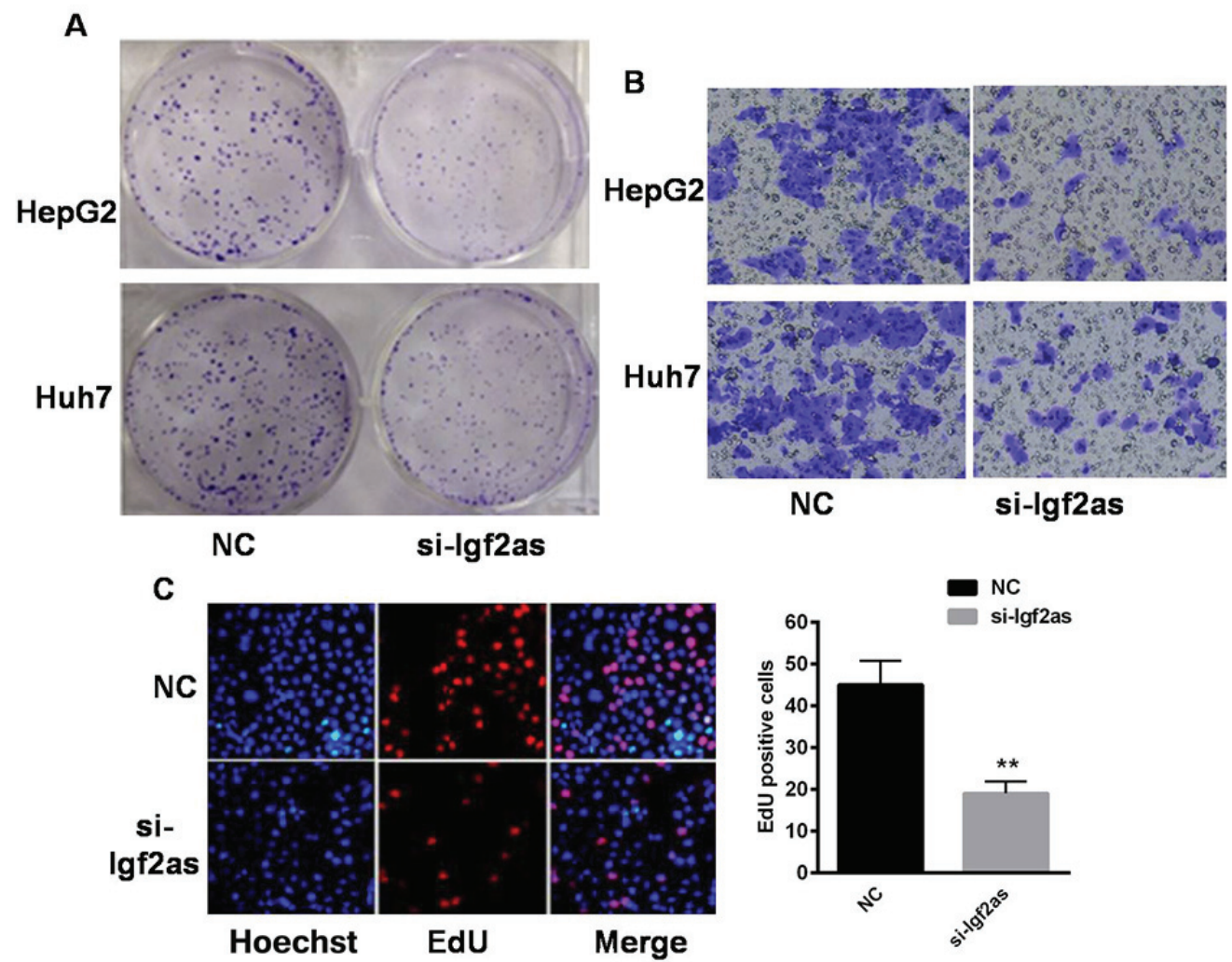

Figure 3. Inhibition of Igf2as reduces colony formation and migration in hepatoma cells. (A) Colony formation was reduced in HepG2 and Huh7 cells transfected with si-Igf2as. (B) Cell invasion was decreased in HepG2 and Huh7 cells transfected with si-Igf2as or NC. (C) HepG2 and Huh7 cell proliferation was evaluated using an EdU incorporation assay. ${ }^{* *} \mathrm{P}<0.01$ vs. the NC. Igf2as, insulin-like growth factor 2 antisense 1; NC, negative control; si-Igfas, small interfering RNA targeting insulin-like growth factor 2 antisense 1; EdU, 5-ethynyl-2'-deoxyuridine.

A
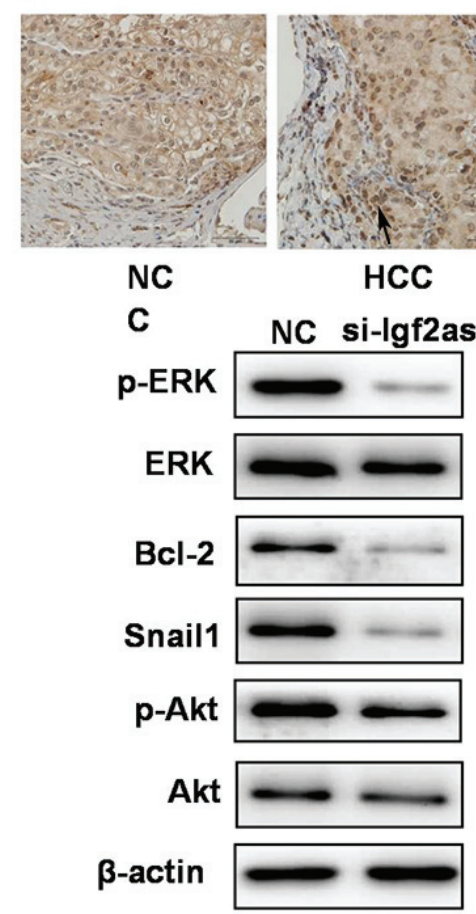

HCC
B

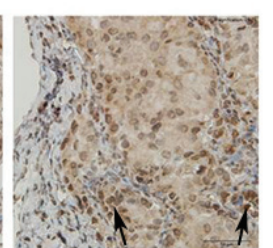

\section{.}

.
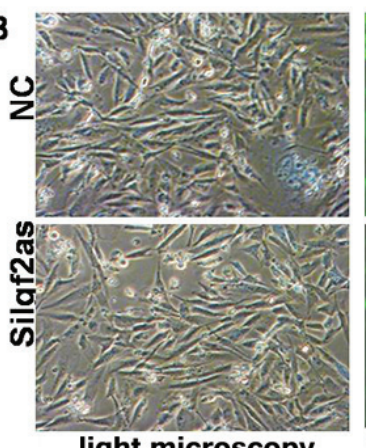

light microscopy
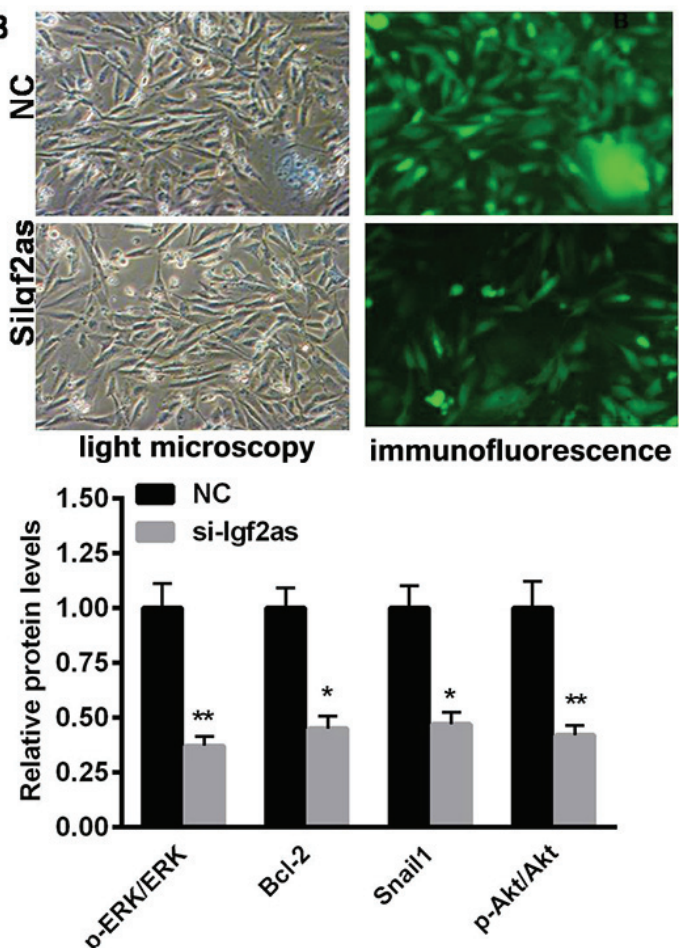

Figure 4. Knockdown of Igf2as reduces ERK activation in HepG2 cells. (A) Immunohistochemistry analysis demonstrated that the phosphorylation of ERK is reduced following knockdown of Igf2as. (B) Inhibition of Igf2as reduced the level of p-ERK. (C) Western blot demonstrating that the level ERK activation and a number of downstream signaling proteins were decreased following treatment with si-Igf2as. (D) Quantification of western blotting revealed that the levels of p-ERK/ERK, Bcl-2, Snal1 and p-Akt/Akt were reduced by Igf2as knockdown. ${ }^{*} \mathrm{P}<0.05,{ }^{* *} \mathrm{P}<0.01$ vs. the NC. Igf2as, insulin-like growth factor 2 antisense 1 ; NC, negative control; si-Igfas, small interfering RNA targeting Igf2as; ERK, extracellular signal-regulated kinase, p-ERK, phosphorylated ERK; Bcl-2, B-cell lymphoma 2; Akt, protein kinase B; p-Akt, phosphorylated Akt; HCC, hepatocellular carcinoma. 
Akt phosphorylation, and the expression of Bcl-2 and Snaill, were also significantly reduced $(\mathrm{P}<0.05 ;$ Fig. $4 \mathrm{C}$ and $\mathrm{D})$. These results suggest that there is a positive correlation between Igf2as expression and ERK activation.

\section{Discussion}

A number of strategies have been developed to improve the survival rate of patients with HCC. However, the mortality of patients with HCC remains high due to poor therapeutic outcomes. Therefore, it is important to investigate novel targets for the treatment of patients with HCC.

lncRNAs are a novel class of non-coding RNAs that are $>200 \mathrm{nt}$ long. Previous studies have demonstrated the important roles that lncRNAs serve in cancer tissues (16-18). Although dysregulated IncRNAs have been implicated in numerous types of cancer, the specific mechanisms underlying their role in these pathologies has yet to be elucidated. For instance, homeotic gene transcript antisense RNA lncRNA has been identified to be significantly upregulated in gastric cancer, and associated with cancer progression and lymph node metastasis (19). In addition, metastasis associated in lung adenocarcinoma transcript 1 (MALAT1) has been identified to be increased in colorectal cancers compared with adjacent normal tissues. Patients with higher MALAT1 expression levels typically demonstrate decreased survival rates (20). Additionally, growth arrest specific 5 lncRNA was demonstrated to markedly upregulated in non-small cell lung cancer, and was associated with tumor growth and invasion (20). However, the distinct role of Igf2as in HCC tissues remains to be elucidated.

The present study demonstrated that the expression of Igf2as is significantly upregulated in HCC tissues compared with normal tissues, and that Igf2as expression was increased in hepatoma cells compared with normal hepatic cells. The knockdown of Igf2as significantly increased HCC cell apoptosis and reduced cell viability. Furthermore, cell migration and invasion were decreased in cells transfected with siRNA targeting Igf2as compared with the NC group. These data suggest that increased Igf2as expression promotes HCC progression.

The ERK/MAPK signaling pathway is known to serve important roles in the progression of cancer through affecting a number of cellular processes, including gene expression, and cell proliferation, apoptosis, migration and invasion $(21,22)$. In the current study, a positive correlation between Igf2as expression and ERK activation was demonstrated. The activation of ERK significantly promotes cell proliferation $(23,24)$. Furthermore, ERK is involved in cancer cell proliferation and apoptosis (25). Therefore, the present study primarily focused on investigating whether ERK activation leads to abnormal cell proliferation and apoptosis, and demonstrated that, compared with normal tissue, ERK activation was increased in HCC tissues. To verify the underlying mechanisms of this association, a knockdown of Igf2as was performed, which significantly promoted apoptosis in HepG2 and Huh7 cells. These data suggest that Igf2as upregulation contributes to hepatoma cell proliferation and invasion via regulation of the ERK signaling pathway.

In conclusion, the current study identified that Igf2as is upregulated in HCC tissues and cells. Notably, the data suggest that Igf2as knockdown significantly reduces cancer cell viability, promotes cancer cell proliferation, and decreases colony formation and cell migration, through regulating the MAPK/ERK signaling pathway. These results indicate that Igf2as functions as a tumor oncogene in the progression of HCC.

\section{References}

1. Acharya SK: Epidemiology of hepatocellular carcinoma in India. J Clin Exp Hepatol 4 (Suppl 3): S27-S33, 2014.

2. Bertagna F, Bertoli M, Bosio G, Biasiotto G, Sadeghi R, Giubbini R and Treglia G: Diagnostic role of radiolabelled choline PET or PET/CT in hepatocellular carcinoma: A systematic review and meta-analysis. Hepatol Int 8: 493-500, 2014.

3. Guan Q, Gu J, Zhang H, Ren W, Ji W and Fan Y: Correlation between vascular endothelial growth factor levels and prognosis of hepatocellular carcinoma patients receiving radiofrequency ablation. Biotechnol Biotechnol Equip 29: 119-123, 2015.

4. Hefaiedh R, Sabbegh M, Ennaifer R, Romdhane H, Ben Nejma H, Belhadj N, Gharbi L and Khalfallah T: Percutaneous treatment versus hepatic resection for the treatment of small hepatocellular carcinoma. Tunis Med 92: 711-716, 2014.

5. Mansourian PG, Yoneda M, Krishna Rao M, Martinez FJ, Thomas E and Schiff ER: Effects of statins on the risk of hepatocellular carcinoma. Gastroenterol Hepatol (N Y) 10: 417-426, 2014.

6. Huang MD, Chen WM, Qi FZ, Xia R, Sun M, Xu TP, Yin L, Zhang EB, De W and Shu YQ: Long non-coding RNA ANRIL is upregulated in hepatocellular carcinoma and regulates cell apoptosis by epigenetic silencing of KLF2. J Hematol Oncol 8: $50,2015$.

7. Xu WH, Zhang JB, Dang Z, Li X, Zhou T, Liu J, Wang DS, Song WJ and Dou KF: Long non-coding RNA URHC regulates cell proliferation and apoptosis via ZAK through the ERK/ MAPK signaling pathway in hepatocellular carcinoma. Int J Biol Sci 10: 664-676, 2014.

8. Ponting CP, Oliver PL and Reik W: Evolution and functions of long noncoding RNAs. Cell 136: 629-641, 2009.

9. Tu ZQ, Li RJ, Mei JZ and Li XH: Down-regulation of long non-coding RNA GAS5 is associated with the prognosis of hepatocellular carcinoma. Int J Clin Exp Pathol 7: 4303-4309, 2014.

10. Chen CL, Tseng YW, Wu JC, Chen GY, Lin KC, Hwang SM and $\mathrm{Hu}$ YC: Suppression of hepatocellular carcinoma by baculovirus-mediated expression of long non-coding RNA PTENP1 and MicroRNA regulation. Biomaterials 44: 71-81, 2015.

11. Hua L, Wang CY, Yao KH, Chen JT, Zhang JJ and Ma WL: High expression of long non-coding RNA ANRIL is associated with poor prognosis in hepatocellular carcinoma. Int J Clin Exp Pathol 8: 3076-3082, 2015.

12. Shi XM and Teng F: Up-regulation of long non-coding RNA Sox 2 ot promotes hepatocellular carcinoma cell metastasis and correlates with poor prognosis. Int J Clin Exp Pathol 8: 4008-4014, 2015

13. Cao Y, Lindström S, Schumacher F, Stevens VL, Albanes D, Berndt S, Boeing H, Bueno-de-Mesquita HB, Canzian F, Chamosa $\mathrm{S}$, et al: Insulin-like growth factor pathway genetic polymorphisms, circulating IGF1 and IGFBP3, and prostate cancer survival. J Natl Cancer Inst 106: dju085, 2014.

14. Vu TH, Chuyen NV, Li T and Hoffman AR: Loss of imprinting of IGF2 sense and antisense transcripts in Wilms' tumor. Cancer Res 63: 1900-1905, 2003.

15. Livak KJ and Schmittgen TD: Analysis of relative gene expression data using real-time quantitative PCR and the 2(-Delta Delta C(T)) method. Methods 25: 402-408, 2001.

16. Malik R, Patel L, Prensner JR, Shi Y, Iyer MK, Subramaniyan S, Carley A, Niknafs YS, Sahu A, Han S, et al: The lncRNA PCAT29 inhibits oncogenic phenotypes in prostate cancer. Mol Cancer Res 12: 1081-1087, 2014.

17. Zhu M, Chen Q, Liu X, Sun Q, Zhao X, Deng R, Wang Y, Huang J, Xu M, Yan J and Yu J: lncRNA H19/miR-675 axis represses prostate cancer metastasis by targeting TGFBI. FEBS J 281: 3766-3775, 2014.

18. Xu C, Shao Y, Xia T, Yang Y, Dai J, Luo L, Zhang X, Sun W, Song H, Xiao B and Guo J: lncRNA-AC130710 targeting by miR-129-5p is upregulated in gastric cancer and associates with poor prognosis. Tumour Biol 35: 9701-9706, 2014. 
19. Endo H, Shiroki T, Nakagawa T, Yokoyama M, Tamai K, Yamanami H, Fujiya T, Sato I, Yamaguchi K, Tanaka N, et al: Enhanced expression of long non-coding RNA HOTAIR is associated with the development of gastric cancer. PLoS One 8: e77070, 2013.

20. Zheng HT, Shi DB, Wang YW, Li XX, Xu Y, Tripathi P, Gu WL, Cai GX and Cai SJ: High expression of lncRNA MALAT1 suggests a biomarker of poor prognosis in colorectal cancer. Int J Clin Exp Pathol 7: 3174-3181, 2014.

21. Chang L and Karin M: Mammalian MAP kinase signalling cascades. Nature 410: 37-40, 2001.
22. Wang X, Lu N, Niu B, Chen X, Xie J and Cheng N: Overexpression of Aurora-A enhances invasion and matrix metalloproteinase-2 expression in esophageal squamous cell carcinoma cells. Mol Cancer Res 10: 588-596, 2012.

23. Kinkade CW, Castillo-Martin M, Puzio-Kuter A, Yan J, Foster TH, Gao H, Sun Y, Ouyang X, Gerald WL, Cordon-Cardo C and Abate-Shen C: Targeting AKT/mTOR and ERK MAPK signaling inhibits hormone-refractory prostate cancer in a preclinical mouse model. J Clin Invest 118: 3051-3064, 2008. 